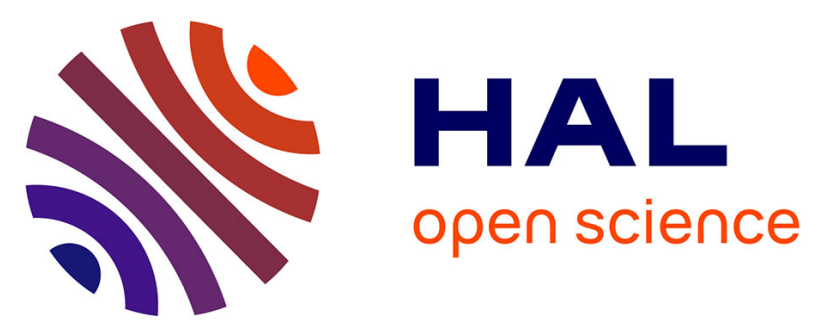

\title{
A Novel Heat Transfer Coefficient Identification Methodology for the Profile Extrusion Calibration Stage
}

Filipe Marques, Stéphane Clain, Gaspar Machado, Bruno Martins, Olga Sousa

Carneiro, Miguel João Nóbrega

\section{To cite this version:}

Filipe Marques, Stéphane Clain, Gaspar Machado, Bruno Martins, Olga Sousa Carneiro, et al.. A Novel Heat Transfer Coefficient Identification Methodology for the Profile Extrusion Calibration Stage. Applied Thermal Engineering, 2016, 103, pp.102-111. 10.1016/j.applthermaleng.2016.04.013 . hal01251310

\author{
HAL Id: hal-01251310 \\ https://hal.science/hal-01251310
}

Submitted on 5 Jan 2016

HAL is a multi-disciplinary open access archive for the deposit and dissemination of scientific research documents, whether they are published or not. The documents may come from teaching and research institutions in France or abroad, or from public or private research centers.
L'archive ouverte pluridisciplinaire HAL, est destinée au dépôt et à la diffusion de documents scientifiques de niveau recherche, publiés ou non, émanant des établissements d'enseignement et de recherche français ou étrangers, des laboratoires publics ou privés.

\section{(ㅇ)(1) $\$$}

Distributed under a Creative Commons Attribution - NonCommerciall 4.0 International 


\title{
A Novel Heat Transfer Coefficient Identification Methodology for the Profile Extrusion Calibration Stage
}

\author{
Filipe Marques ${ }^{\mathrm{a}}$, Stéphane Clain ${ }^{\mathrm{a}, \mathrm{c}}$, Gaspar J. Machado ${ }^{\mathrm{a}}$, Bruno Martins ${ }^{\mathrm{b}}$, Olga S. \\ Carneiro $^{b}$, João M. Nóbrega ${ }^{b}$ \\ ${ }^{a}$ Centre of Mathematics, University of Minho, Campus de Azurém, 4800-058 Guimarães, Portugal \\ e-mail: a62055@alunos.uminho.pt,\{clain,gjm\}@math.uminho.pt \\ ${ }^{b}$ Institute for Polymers and Composites/I3N, University of Minho, Campus de Azurém, 4800-058 \\ Guimarães, Portugal \\ e-mail: brunodiogomartins22@gmail.com,\{olgasc,mnobrega\}@dep.uminho.pt \\ ${ }^{c}$ Institut de Mathématiques de Toulouse, Université Paul Sabatier, 31062 Toulouse, France
}

\begin{abstract}
A new method to compute heat transfer coefficients of the profile extrusion process calibration stage, in conjunction with a prototype calibration system [1, is proposed. The methodology involves two major ingredients: a numerical modeling code and a fitting procedure. The code, based on the Finite Volume Method, computes the steadystate solution for the heat transfer problem. The software carefully handles discontinuous solutions as well as discontinuities of the velocity and the material characteristics. Fitting procedure introduces alternative algorithms we have tested and assessed in [2. A real case study demonstrates the advantages of using the new proposed methodology when compared with the previously applied [1].
\end{abstract}

Keywords: Profile extrusion cooling stage, heat transfer coefficient identification, fitting, finite volume method, polymer flow

\section{Introduction}

As in other industrial areas, the numerical simulation of the extrusion process is a fundamental tool to support the design and development of extruders, extrusion dies, and calibration/cooling systems, in order to optimize the production rate while providing high quality products [3, 4. Basically, in the case of thermoplastics extrusion, the numerical model consists of coupling the Navier-Stokes equations for non-Newtonian material and the non-linear convection-diffusion equation for the thermal process, or the Stefan equation when a phase change occurs. When the extrusion of thermoplastics profile is considered, specific difficulties arise in the numerical simulation of the heat transfer that holds at the calibration/cooling unit. In fact, special attention has to be paid to the interface between the polymer and the calibrator where, due to the non-perfect contact between the two physical domains, a temperature discontinuity exists. Numerical methods such as the finite element [5, 6, 7, 8, or finite difference hardly provide a good approximation of the cooling process since they require continuity across the contact boundary. Moreover, such methods do not guarantee local energy conservation at the 
cell level and may lead to unrealistic evaluation of the heat transfer process. The finite volume method is a popular technique due to its built-in conservative property 9 . Its simplicity and versatility turns the method to be very competitive when compared with the traditional finite element method 10, 11, 12 or its recent extension, the finite pointset method [13, 14. Many practical problems in physics and engineering are now discretized using this robust technique on unstructured meshes, and recent progress enables to consider a wide range of applications for two and three dimensional domains. In particular, the finite volume method easily handles discontinuous solutions or discontinuous material coefficients, while still preserving both the local energy conservation and the accuracy.

Increasing the production rate and the quality of the produced profiles are two antagonistic goals, since the increase of the production speed generally leads to a decrease in the product quality. The cooling stage is critical since it generally determines the production rate, i.e. it is the limiting stage of the extrusion process, and may have a strong influence on the profile quality as it determines the degree of residual stresses present in the final product. The low thermal diffusivity of thermoplastics is the major reason for that behavior. On one hand it slows down the profile cooling process, demanding long residence times in the cooling unit, and, on the other hand it is responsible for the development of huge thermal gradients that give rise to internal residual stresses, thus decreasing the profile performance. Consequently, the cooling stage has a crucial role since solidification of the profile outer layers should be as fast as possible and, at the same time, temperature non-homogeneity should be minimized. To circumvent this problem, the authors of this work have been developing several numerical codes to deal with the heat transfer at the calibration/cooling unit, and concluded that one of the most influential parameters, required as input in the numerical codes, is the heat transfer coefficient at the polymer-calibrator interface, $h_{\text {int }}$. In turn, this coefficient highly depends on process conditions (such as the roughness of the calibration material, the extrusion velocity, and the degree of vacuum, among others) and may vary within a wide range 11. Therefore, in the simulations of the calibration/cooling stage, accurate values of $h_{\text {int }}$, determined in well controlled conditions, should be considered. For this sake, a prototype calibration/cooling system, previously presented in 1, was developed, being the numerical codes developed for simulation purposes also used to compute, by inverse engineering, $h_{\text {int }}$. However, the determination of this coefficient resorting to the referred prototype faces some other difficulties since an accurate model and numerical scheme are mandatory to provide approximations leading to a correct valuation of this coefficient [15]. In [16], it is shown that the generic second-order finite volume scheme for the convection-diffusion-reaction equation based on the cell to vertex technology is very efficient and robust. In [17] the scheme is tested for non-homogeneous and anisotropic problems.

Furthermore, the determination of $h_{\text {int }}$ requires the introduction, as input variables, of some quantities that are not known with enough accuracy. Those variables are the convection heat transfer coefficient of the air, $h_{\text {air }}$, the polymer inflow temperature (i.e. polymer temperature at the extrusion die outlet), $T_{\mathrm{in}}$, and the air temperature, $T_{\text {air }}$ (see Fig. 1(a)). Bearing this in mind, to correctly compute the values of the aforementioned parameters during the experimental determination of $h_{\text {int }}$, the temperature of the extruded tape should be measured in several locations. The objective is to minimize the error associated with the $h_{\text {int }}$ value, through the use of a function that will opti- 
mize, simultaneously, the values of all the unknowns $\left(h_{\mathrm{int}}, h_{\mathrm{air}}, T_{\mathrm{int}}\right.$, and $\left.T_{\text {air }}\right)$ in the reverse mode usage of the heat transfer numerical code. It should be noted that the uncertainty of the values of the referred variables $\left(h_{\text {air }}, T_{\text {in }}\right.$, and $\left.T_{\text {air }}\right)$ are expected to have a much higher impact in the determination of $h_{\text {int }}$ than in the simulation of the calibration/cooling stage.

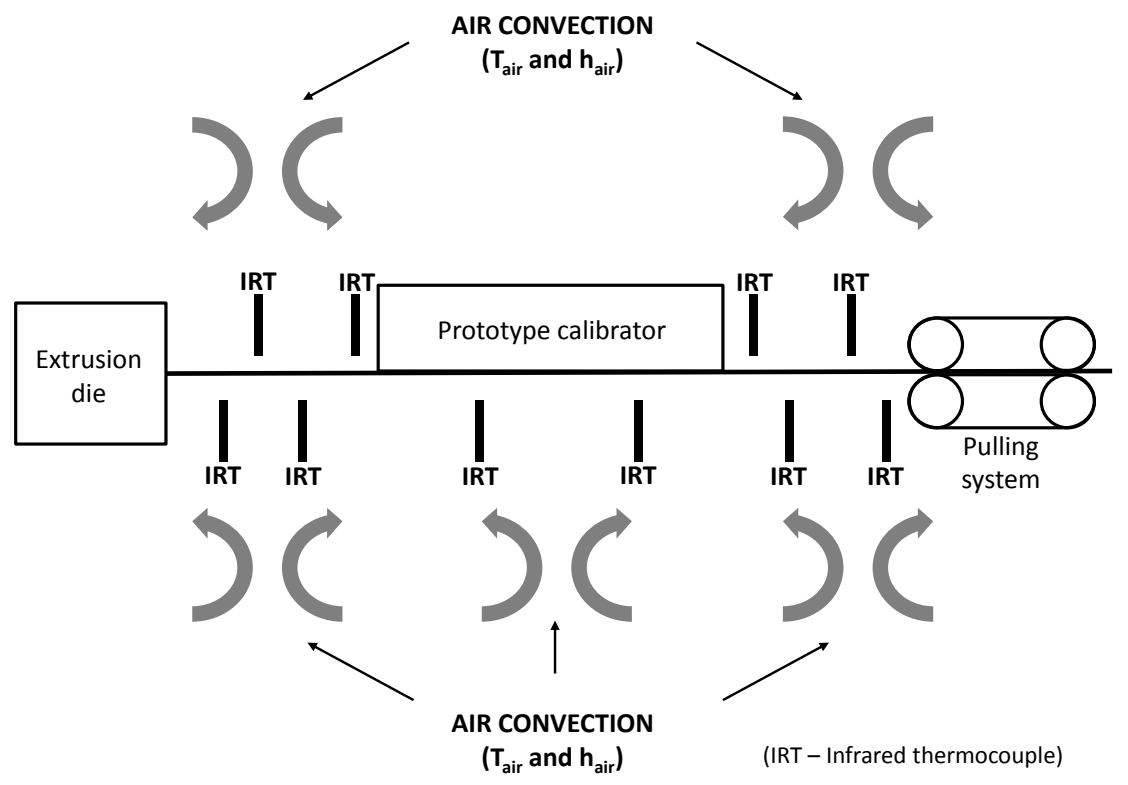

(a)

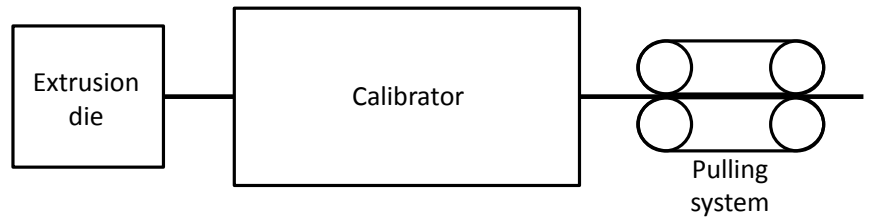

(b)

Figure 1: Calibration stage schematic layout:(a) prototype developed to characterize $h_{\text {int }}$; (b) Typical extrusion line.

In fact, in the prototype calibrator used to determine $h_{\text {int }}$ the extruded polymer tape is exposed to environmental conditions during the entire path (both surfaces before and after the prototype, and the lower surface during calibration), as can be seen in Fig. 11(a). Also, the exposed length is higher to enable the placement of several infrared thermocouples for the measurement of its temperature at several locations. In a real calibration unit, environmental conditions will only affect the (short) tape length outside the calibrator, as illustrated in Fig. 1(b). Thus, in this case the majority of the heat transfer occurs inside the calibrator, where the effect of $h_{\text {int }}$ is dominant and much more intensive than that of $h_{\text {air. }}$. To sum up, in the present work, we aim to develop an algorithm for the 
simultaneous optimization of several parameters of the heat transfer in profile extrusion calibration by employing a method for discontinuous situations, both for the solution and the material coefficients. The study considers an aluminium prototype calibrator cooling a polymer (Polystyrene) tape, where we seek for an accurate optimization of the unknown parameters, based on data collected in extrusion runs performed with the referred prototype [1, with a view to determine accurate values for the heat transfer coefficient at the polymer-calibrator interface.

\section{The process modelling}

As referred in the previous section and illustrated in Fig. 1. (b), in a profile extrusion line the polymer profile passes through a calibrator after leaving the extrusion die. The calibrator, as schematized in Fig. 2, is a metallic tool maintained at a low temperature via the circulation of a cold fluid in its cooling channels. It encompasses also several slots through which vacuum is applied to assure the contact between the hot polymer profile and the cold calibration surface. The heat transfer process occurs essentially in the path through the calibrator, and also (at a minor extent) in the air path before and after the calibrator.

In the scope of this work, we consider the multi-domain polymer-calibrator system, represented in Fig. 3 and constituted of two subdomains where $\Omega_{\mathrm{c}}$ and $\Omega_{\mathrm{p}}$ stand for the calibrator and polymer (the subscripts $\mathrm{c}$ and $\mathrm{p}$ refer to the calibrator and polymer, respectively) while the boundaries are defined as:

- calibrator: $\Gamma_{\mathrm{c}}=\Gamma_{\text {sup }} \cup \Gamma_{\text {lat }} \cup \Lambda$,

- polymer: $\Gamma_{\mathrm{p}}=\Gamma_{\text {air }} \cup \Gamma_{\text {in }} \cup \Gamma_{\text {out }} \cup \Lambda$.

\subsection{Heat transfer problem}

A two-dimensional convection-diffusion model based on the energy conservation equations for the two domains is used to model the flow and the extruder, namely

$$
\begin{aligned}
& \nabla \cdot\left(C_{\mathrm{p}} U_{\mathrm{p}} T_{\mathrm{p}}-k_{\mathrm{p}} \nabla T_{\mathrm{p}}\right)=0 \quad \text { in } \Omega_{\mathrm{p}} \\
& -\nabla \cdot\left(k_{\mathrm{c}} \nabla T_{\mathrm{c}}\right)=0 \quad \text { in } \Omega_{\mathrm{c}},
\end{aligned}
$$

where $k_{\mathrm{p}}$ and $k_{\mathrm{c}}$ are the thermal conductivity of the polymer and the calibrator respectively, $T_{\mathrm{p}}$ and $T_{\mathrm{c}}$ represent the temperature distribution in the polymer and the calibrator respectively, and $U_{\mathrm{p}}$ and $C_{\mathrm{p}}$ are the velocity and the volumetric heat capacity of the polymer respectively. We then prescribe the following boundary conditions.

- The heat transfer between the polymer and the calibrator is defined as a thermal contact resistance, which originates a discontinuity in the temperature domain. The energy flux across the interface is assumed to be linear with respect to the difference of the temperatures given by

$$
k_{\mathrm{c}} \frac{\partial T_{\mathrm{c}}}{\partial n_{\mathrm{c}}}=-k_{\mathrm{p}} \frac{\partial T_{\mathrm{p}}}{\partial n_{\mathrm{p}}}=h_{\mathrm{int}}\left(T_{\mathrm{p}}-T_{\mathrm{c}}\right) \quad \text { on } \Lambda,
$$

where $n_{\mathrm{p}}$ and $n_{\mathrm{c}}$ denote the unit normal vector to the boundaries of $\Omega_{\mathrm{p}}$ and $\Omega_{\mathrm{c}}$ respectively. 


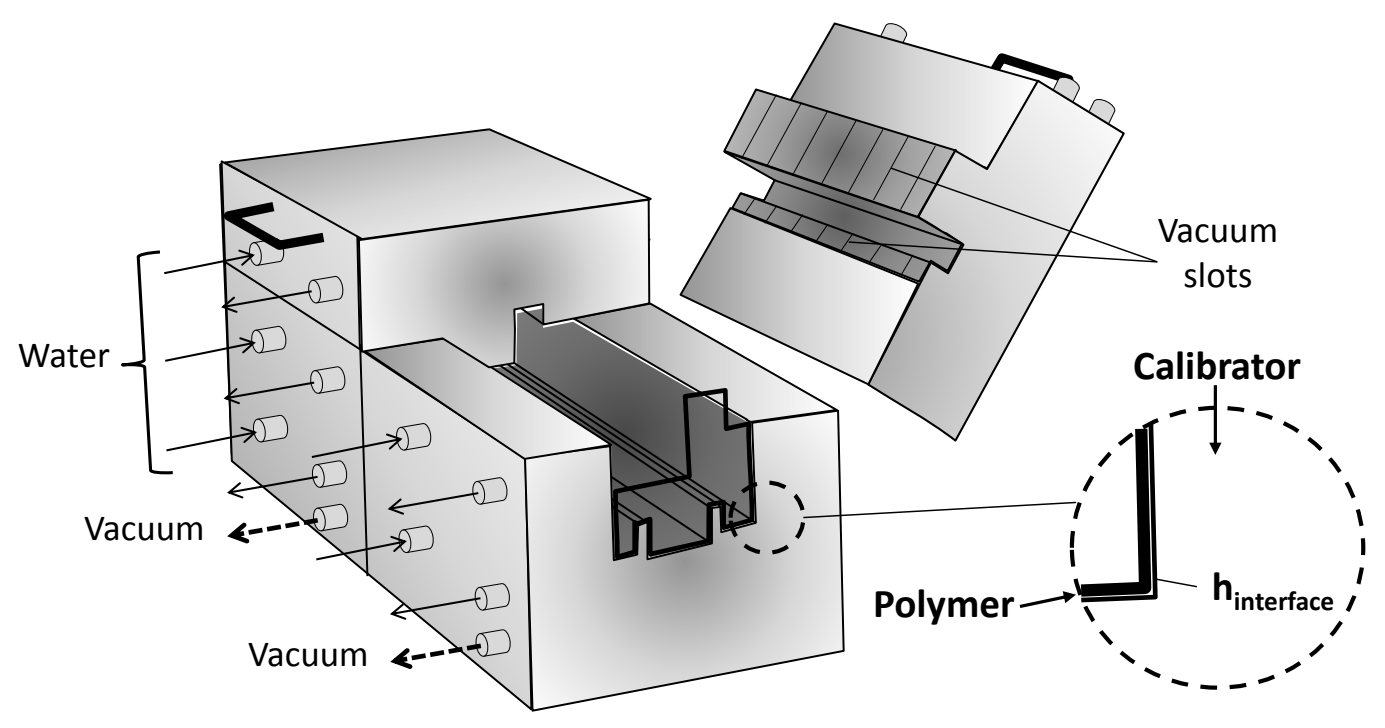

Figure 2: Typical calibration unit for profiles.

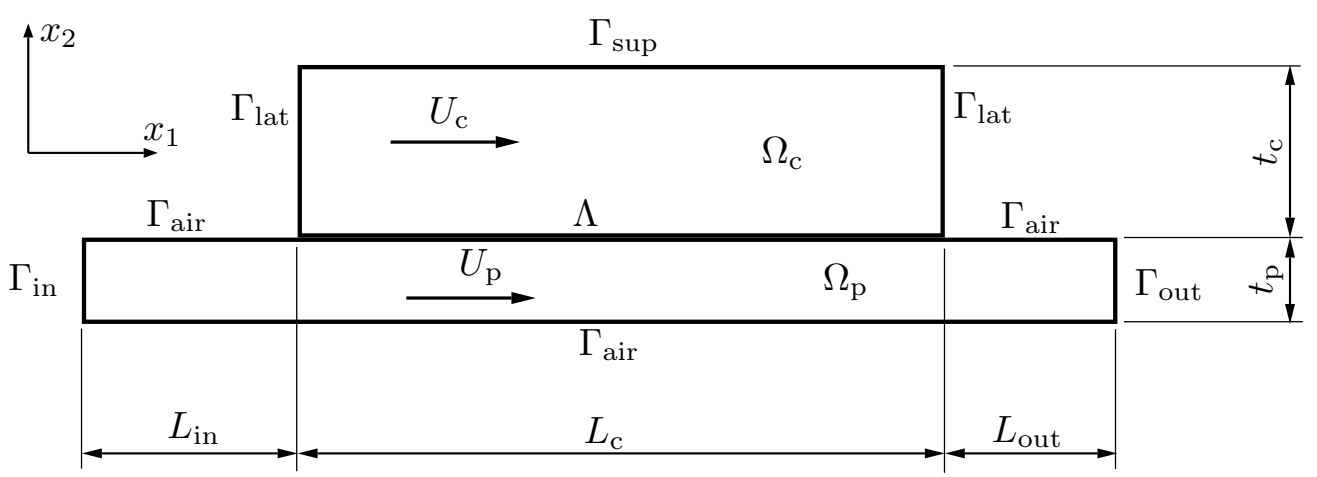

Figure 3: Two-dimensional geometry used to model the heat transfer between the calibrator and the polymer. 
- The temperature distribution on $\Gamma_{\text {sup }}$ is a given polynomial function $T_{\text {sup }}=T\left(x_{1}\right)$, experimentally determined with the data provided by thermocouples embedded in the calibrator block [1].

- On the lateral sides of the calibrator, the bottom and the upper surfaces, natural convection is assumed

$$
-k_{\mathrm{c}} \frac{\partial T_{\mathrm{c}}}{\partial n_{\mathrm{c}}}=h_{\mathrm{air}}\left(T_{\mathrm{c}}-T_{\text {air }}\right) \quad \text { on } \Gamma_{\text {lat }} \cup \Gamma_{\text {air }},
$$

where $T_{\text {air }}$ denotes the temperature of the air which is considered a constant value, and $h_{\text {air }}$ is the natural convection heat transfer coefficient.

- For the polymer inflow left boundary, we assume a constant prescribed temperature $T=T_{\text {in }}$ on $\Gamma_{\text {in }}$ which corresponds to the extrusion temperature, whereas we assume an adiabatic condition for the polymer outflow on the right boundary

$$
-k_{\mathrm{p}} \frac{\partial T_{\mathrm{p}}}{\partial n_{\mathrm{p}}}=0 \quad \text { on } \Gamma_{\text {out }} .
$$

\section{Finite volume scheme}

We provide a short description of the finite volume scheme based on the cell-to-vertex technology presented in [16, that closely follows the scheme proposed in 2. We consider the energy equation on an open bounded polygonal domain $\Omega$ of $\mathbb{R}^{2}$ with boundary $\Gamma$ (we skip the index p for the sake of simplicity). We seek the temperature distribution $T \equiv T\left(x_{1}, x_{2}\right)$ as a solution of the steady-state convection-diffusion equation

$$
\nabla \cdot(V T-k \nabla T)=0 \quad \text { in } \Omega,
$$

where $V T$ is the convective term with $V=C U$ and $k \nabla T$ is the diffusive term. The boundary of the domain is partitioned into four subsets $\Gamma_{\mathrm{D}}, \Gamma_{\mathrm{N}}, \Gamma_{\mathrm{R}}$, and $\Lambda$ where different types of conditions may be prescribed, namely:

- Dirichlet: $T=T_{\mathrm{D}}$ on $\Gamma_{\mathrm{D}}$;

- Neumann (adiabatic): $-k \nabla T \cdot n=0$ on $\Gamma_{\mathrm{N}}$;

- interface polymer-air: $(V T-k \nabla T) \cdot n=h\left(T-T_{\text {air }}\right)$ on $\Gamma_{\mathrm{R}}$.

- interface polymer-calibrator: $k_{\mathrm{c}} \nabla T_{\mathrm{c}} \cdot n_{\mathrm{c}}=-k_{\mathrm{p}} \nabla T_{\mathrm{p}} \cdot n_{\mathrm{p}}=h\left(T_{\mathrm{p}}-T_{\mathrm{c}}\right)$ on $\Lambda$.

\subsection{Mesh and notations}

We denote by $\mathcal{T}$ a mesh consisting of $I$ non-overlapping convex polygonal cells $c_{i}$, $i=1, \ldots, I$, and $K$ vertices $v_{k}, k=1, \ldots, K$. We highlight that $\mathcal{T}$ composes two submeshes, $\mathcal{T}_{\mathrm{c}}$ and $\mathcal{T}_{\mathrm{p}}$, for the subdomains $\Omega_{\mathrm{c}}$ and $\Omega_{\mathrm{p}}$, respectively, and are conformed with $\Lambda$ such that if $\stackrel{\circ}{\cap} \Lambda \neq \varnothing$ then $e \subset \Lambda$. We adopt the following conventions (see Fig. 4p:

- the mesh $\mathcal{T}_{\mathrm{c}}$ consists of $I_{\mathrm{c}}$ non-overlapping convex polygonal cells $c_{i_{\mathrm{c}}}, i_{\mathrm{c}}=1, \ldots, I_{\mathrm{c}}$, and $K_{\mathrm{c}}$ vertices $v_{k_{\mathrm{c}}}, k_{\mathrm{c}}=1, \ldots, K_{\mathrm{c}}$; 
- the mesh $\mathcal{T}_{\mathrm{p}}$ consists of $I_{\mathrm{p}}$ non-overlapping convex polygonal cells $c_{i_{\mathrm{p}}}, i_{\mathrm{p}}=I_{\mathrm{c}}+$ $1, \ldots, I_{\mathrm{c}}+I_{\mathrm{p}}$, and $K_{\mathrm{p}}$ vertices $v_{k_{\mathrm{p}}}, k_{\mathrm{p}}=K_{\mathrm{c}}+1, \ldots, K_{\mathrm{c}}+K_{\mathrm{p}}$;

- for any cell $c_{i}, \partial c_{i}$ represents its boundary and $\left|c_{i}\right|$ its area; we denote by $m_{i}$ the mass centre of $c_{i}$;

- two cells $c_{i}$ and $c_{j}$ share a common edge $e_{i j}$ whose length is $\left|e_{i j}\right|$ and the midpoint is $m_{i j} ; n_{i j}$ is the unit normal vector to $e_{i j}$ outward to $c_{i}$, i.e. $n_{i j}=-n_{j i}$; if an edge of $c_{i}$ belongs to the boundary $\Gamma$, we replace the index $j$ by D, N, R, or $\Lambda$ if $e_{i j}$ belongs to $\Gamma_{\mathrm{D}}, \Gamma_{\mathrm{N}}, \Gamma_{\mathrm{R}}$, or $\Lambda$, respectively;

- for any cell $c_{i}$ belonging to $\Omega_{\mathrm{c}}$ or $\Omega_{\mathrm{p}}$ we associate the index set $\nu\left(i_{\mathrm{c}}\right) \subset\left\{1, \ldots, I_{\mathrm{c}}\right\} \cup$ $\{\mathrm{D}, \mathrm{N}, \mathrm{R}, \Lambda\}$ or $\nu\left(i_{\mathrm{p}}\right) \subset\left\{I_{\mathrm{c}}+1, \ldots, I_{\mathrm{c}}+I_{\mathrm{p}}\right\} \cup\{\mathrm{D}, \mathrm{N}, \mathrm{R}, \Lambda\}$, respectively, such that $j \in \nu(i)$ if $e_{i j}$ is a common edge of $c_{i}$ and $c_{j}$ or with the boundary $\Gamma_{j}$ if $j=\{\mathrm{D}, \mathrm{N}, \mathrm{R}\}$ or with the interface $\Lambda$;

- for any vertex $v_{k}$ belonging to $\Omega_{\mathrm{c}}$ or $\Omega_{\mathrm{p}}$ we associate the index set $\mu\left(k_{\mathrm{c}}\right) \subset$ $\left\{1, \ldots, I_{\mathrm{c}}\right\}$ or $\mu\left(k_{\mathrm{p}}\right) \subset\left\{I_{\mathrm{c}}+1, \ldots, I_{\mathrm{c}}+I_{\mathrm{p}}\right\}$, respectively, such that $i \in \mu(k)$ if $v_{k}$ is a vertex belonging to the cell $c_{i}$.

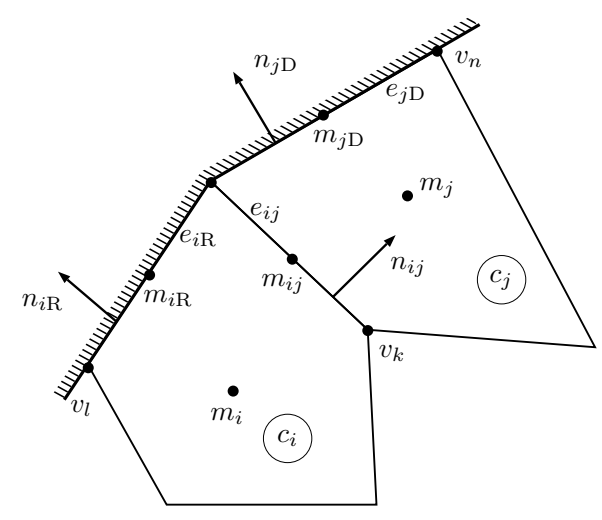

Figure 4: Mesh notation.

\subsection{Second-order scheme}

To provide the finite volume scheme, Eq. (1) should be integrated over cell $c_{i}$

$$
\int_{c_{i}} \nabla \cdot(V T-k \nabla T) \mathrm{d} x=0,
$$

and applying the divergence theorem we get an integral over the surface

$$
\int_{\partial c_{i}}(V T-k \nabla T) \cdot n \mathrm{~d} s=0,
$$

and, then,

$$
\sum_{j \in \nu(i)} \int_{e_{i j}}\left(V \cdot n_{i j} T-k \nabla T \cdot n_{i j}\right) \mathrm{d} s=0 .
$$


The numerical integration of Eq. (2), based on a quadrature method, introduces consistency second-order errors and provides the following approximation

$$
\sum_{j \in \nu(i)}\left|e_{i j}\right|\left(V\left(m_{i j}\right) \cdot n_{i j} T\left(m_{i j}\right)-k\left(m_{i j}\right) \nabla T\left(m_{i j}\right) \cdot n_{i j}\right) \approx 0 .
$$

The residual is a function of the vector $\mathbf{T}$ given by

$$
\mathcal{G}_{i}(\mathbf{T})=\sum_{j \in \nu(i)}\left|e_{i j}\right| \mathcal{F}_{i j}(\mathbf{T})
$$

where $\mathcal{F}_{i j}$ is an approximation of the convective and diffusive fluxes through the edge $e_{i j}$ presented in Eq. (3).

\subsection{Flux computation}

Cell centred finite volume methods use the cell centre as collocation points for the unknowns. Nevertheless, the computation of the gradients require an evaluation of the temperature at the vertices. The cell-to-vertex interpolation provides a robust and accurate mechanism to compute the temperature at vertices from the temperature at the cell centres. Let $T_{i}$ be an approximation of $T$ at $m_{i}$. We gather all the approximations in two vectors $\mathbf{T}_{\mathrm{c}}=\left(T_{i}\right)_{i=1, \ldots, I_{\mathrm{c}}}$ and $\mathbf{T}_{\mathrm{p}}=\left(T_{i}\right)_{i=I_{\mathrm{c}}+1, \ldots, I_{\mathrm{c}}+I_{\mathrm{p}}}$ for the calibrator and the polymer domain. Similarly, let $\boldsymbol{\Theta}_{\mathrm{c}}=\left(\theta_{k_{\mathrm{c}}}\right)_{k_{\mathrm{c}}=1, \ldots, K_{\mathrm{c}}}$ and $\boldsymbol{\Theta}_{\mathrm{p}}=\left(\theta_{k_{\mathrm{p}}}\right)_{k_{\mathrm{p}}=K_{\mathrm{c}}+1, \ldots, K_{\mathrm{c}}+K_{\mathrm{p}}}$ be the vectors with the approximations of the temperature at the vertices. We also consider the global vectors $\mathbf{T}=\left(\mathbf{T}_{\mathrm{c}}, \mathbf{T}_{\mathrm{p}}\right)^{t}$ and $\boldsymbol{\Theta}=\left(\boldsymbol{\Theta}_{\mathrm{c}}, \boldsymbol{\Theta}_{\mathrm{p}}\right)^{t}$ corresponding to the temperature distribution in the whole domain associated to the cells and to the vertices respectively.

The linear mapping $\mathbf{T} \rightarrow \boldsymbol{\Theta}(\mathbf{T})$ given by

$$
\theta_{k}=\sum_{i \in \mu(k)} \beta_{k i} T_{i}
$$

with $\beta_{k i}$ the interpolation coefficients for vertex $k$ provides an expression of the temperature at the vertex $v_{i}$ in function of the temperature in the cell $c_{i}, i \in \mu(k)$. Special attention is required when dealing with the discontinuity at the contact interface and two distinct temperatures have to be evaluated at the same vertex (see [2] for a detailed description of the method). The numerical fluxes are then evaluated based on the two vectors $\mathbf{T}$ and $\boldsymbol{\Theta}$.

- For each cell $c_{i}$ we define the affine function

$$
\widetilde{T}_{i}\left(x_{1}, x_{2}\right)=T_{i}+\widetilde{\mathcal{C}}_{i, 1}\left(x_{1}-m_{i, 1}\right)+\widetilde{\mathcal{C}}_{i, 2}\left(x_{2}-m_{i, 2}\right),
$$

where $\widetilde{\mathcal{C}}_{i, 1}$ and $\widetilde{\mathcal{C}}_{i, 2}$ are the coefficients that minimize a quadratic functional and correspond to the best approximation in the least squares sense of the temperature $\theta_{k}$ at the vertices of the cell.

- Each inner edge holds two different polynomials $\widetilde{T}_{i j}$ and $\widetilde{T}_{j i}$ given by

$$
\widetilde{T}_{i j}\left(x_{1}, x_{2}\right)=T_{i}+\widetilde{\mathcal{C}}_{i j, 1}\left(x_{1}-m_{i, 1}\right)+\widetilde{\mathcal{C}}_{i j, 2}\left(x_{2}-m_{i, 2}\right),
$$


and the symmetric expression for $\widetilde{T}_{j i}$, where $\widetilde{\mathcal{C}}_{i j, 1}$ and $\widetilde{\mathcal{C}}_{i j, 2}$ are the unique coefficients that the resultant polynomial interpolates $\theta_{k}$, associated to the two extremities $v_{k}$ of $e_{i j}$. The polynomial associated to edge $e_{i j}$ then writes

$$
\breve{T}_{i j}=\breve{T}_{j i}=\frac{\left|c_{i}\right|}{\left|c_{i}\right|+\left|c_{j}\right|} \widetilde{T}_{i j}+\frac{\left|c_{j}\right|}{\left|c_{i}\right|+\left|c_{j}\right|} \widetilde{T}_{j i}
$$

Denoting $[z]^{+}=\max (0, z)$ and $[z]^{-}=\min (0, z)$, the numerical fluxes should take into account four different situations:

- for an inner edge $e_{i j}$ as

$$
\mathcal{F}_{i j}=\left[V\left(m_{i j}\right) \cdot n_{i j}\right]^{+} \widetilde{T}_{i}\left(m_{i j}\right)+\left[V\left(m_{i j}\right) \cdot n_{i j}\right]^{-} \widetilde{T}_{j}\left(m_{i j}\right)-k\left(m_{i j}\right) \nabla \check{T}_{i j}\left(m_{i j}\right) \cdot n_{i j}
$$

- for a Dirichlet boundary edge $e_{i \mathrm{D}}$ as

$$
\mathcal{F}_{i \mathrm{D}}=\left[V\left(m_{i \mathrm{D}}\right) \cdot n_{i \mathrm{D}}\right]^{+} \widetilde{T}_{i}\left(m_{i \mathrm{D}}\right)+\left[V\left(m_{i \mathrm{D}}\right) \cdot n_{i \mathrm{D}}\right]^{-} T_{\mathrm{D}}\left(m_{i \mathrm{D}}\right)-k\left(m_{i \mathrm{D}}\right) \nabla \widetilde{T}_{i \mathrm{D}}\left(m_{i \mathrm{D}}\right) \cdot n_{i \mathrm{D}} ;
$$

- for a Neumann boundary edge $e_{i \mathrm{~N}}$ as

$$
\mathcal{F}_{i \mathrm{P}}=V\left(m_{i \mathrm{~N}}\right) \cdot n_{i \mathrm{~N}} \widetilde{T}_{i}\left(m_{i \mathrm{~N}}\right)
$$

- for a heat transfer boundary edge $e_{i \mathrm{R}}$ as

$$
\mathcal{F}_{i \mathrm{R}}=h\left(\widetilde{T}_{i}\left(m_{i \mathrm{R}}\right)-T_{\mathrm{R}}\left(m_{i \mathrm{R}}\right)\right) .
$$

In the case of the interface between the polymer and the calibrator, we have to distinguish two cases regarding to the side where the flux comes from (see [2] for the details).

Since $\mathcal{F}_{i j}$ is linearly dependent on vector $\mathbf{T}$, we define the affine operator $\mathbf{T} \rightarrow$ $\mathcal{G}_{i}(\mathbf{T})$ for each cell $c_{i}, i=1, \ldots, I$. Gathering all the components $\mathcal{G}_{i}(\mathbf{T})$ of the residual in vector $\mathcal{G}(\mathbf{T})$, we seek the solution vector $\mathbf{T}^{\star}$ such that $\mathcal{G}\left(\mathbf{T}^{\star}\right)=0_{I}$. We obtain a matrix-free scheme and the affine problem is solved by applying a preconditioned GMRES procedure.

\section{Parameter identification procedure}

We assume that the cooling process is mainly governed by five parameters: the heat transfer coefficients $h_{\text {int }}$ and $h_{\text {air }}$, the temperatures $T_{\text {air }}$ and $T_{\text {in }}$, and the velocity $u$. In practice, some coefficients are measured (temperature, velocity) while the other parameters have to be deduced from the experimental tests. To this end, several sensors measure the temperature $\hat{T}_{\mathrm{p}_{\ell}}$ at points $q_{\ell}, \ell=1, \ldots, C_{\ell}$, and the goal is to identify the set of parameters

$$
\mathbf{H}=\left(H_{1}, \ldots, H_{m}\right)
$$

that provides the polymer temperature approximation $T_{\mathrm{p}}^{\mathbf{H}}$ that better suits the experimental measurements. More precisely, we seek the set of $m$ parameters $\mathbf{H}$ that minimize 
the error between the measured temperatures and its the numerical approximation given by the following functional

$$
F(\mathbf{H})=\sum_{\ell=1}^{C_{\ell}}\left[T_{\mathrm{p}}^{\mathbf{H}}\left(q_{\ell}\right)-\hat{T}_{\mathrm{p}_{\ell}}\right]^{2},
$$

where $C_{\ell}$ is the number of sensors.

Notice that the set of parameters $\mathbf{H}$ may be different from an operation to another one in function of the experimental conditions. Indeed, one can consider a problem with two parameters as $\mathbf{H}=\left(h_{\text {int }}, h_{\text {air }}\right)$ if the others parameters are given or a problem with four parameters as $\mathbf{H}=\left(h_{\mathrm{int}}, h_{\text {air }}, T_{\text {air }}, T_{\text {in }}\right)$ when the temperatures also are unknown.

To provide the optimal set of parameters, an iterative procedure is considered where a variation $\Delta \mathbf{H}^{n}$ to the parameters $\mathbf{H}^{n}$ provides a better approximation $\mathbf{H}^{n+1}=\mathbf{H}^{n}+$ $\Delta \mathbf{H}^{n}$ such that $F\left(\mathbf{H}^{n+1}\right)<F\left(\mathbf{H}^{n}\right)$. Several optimization techniques will be considered to achieve this goal.

\subsection{The Newton-Raphson method}

The Newton-Raphson technique is a generic procedure to provide the zeros of a vector-valued function using successive linear approximations. Since we want to minimize function $F$, we seek vector $\mathbf{H}$ such that $\nabla F(\mathbf{H})=\mathbf{0}$. We then introduce

$$
\Delta \mathbf{H}=-\left[\nabla^{2} F(\mathbf{H})\right]^{-1} \nabla F(\mathbf{H}),
$$

where $\nabla F$ is the gradient vector and $\nabla^{2} F$ denotes the Hessian matrix.

To evaluate the derivatives, the finite differences were adopted. We define a tolerance value $\varepsilon$ and set

$$
\varepsilon_{\alpha}=H_{\alpha} \varepsilon, \quad \alpha=1, \ldots, m .
$$

An approximation of the first derivatives then writes

$$
\frac{\partial F}{\partial H_{i}} \approx \frac{F\left(H_{i}+\varepsilon_{i}, \ldots\right)-F\left(H_{i}-\varepsilon_{i}, \ldots\right)}{2 \varepsilon_{i}}
$$

and we proceed in a similar way to evaluate approximations for the second derivative $\frac{\partial^{2} F}{\partial H_{i} \partial H_{j}}$ involved in the Hessian matrix.

Notice that the optimization procedure requires to evaluate $1+2 m^{2}$ times the function $F$ in each iteration, which means that the thermal problem has to be solved the same amount of times leading to an important and unnecessary computational effort.

\subsection{The Gauss-Newton method}

The Gauss-Newton method is a specific modification of the Newton-Raphson method for nonlinear least squares problems. The main advantage is that much less computational effort is required since it avoids the calculation of second derivatives.

The least squares problem given by Eq. (4) can be rewritten in vectorial form as

$$
F(\mathbf{H})=\left(\mathbf{T}_{\mathrm{p}}^{\mathbf{H}}-\hat{\mathbf{T}}_{\mathrm{p}}\right)^{T}\left(\mathbf{T}_{\mathrm{p}}^{\mathbf{H}}-\hat{\mathbf{T}}_{\mathrm{p}}\right) .
$$


In order to evaluate the temperature distribution in the neighborhood of $\mathbf{H}$, we consider the first-order Taylor series expansion, yielding

$$
\mathbf{T}_{\mathrm{p}}^{\mathbf{H}+\Delta \mathbf{H}} \approx \mathbf{T}_{\mathrm{p}}^{\mathbf{H}}+\frac{\partial \mathbf{T}_{\mathrm{p}}^{\mathbf{H}}}{\partial \mathbf{H}} \Delta \mathbf{H}=\mathbf{T}_{\mathrm{p}}^{\mathbf{H}}+\mathbf{J} \Delta \mathbf{H}
$$

where $\frac{\partial \mathbf{T}_{\mathbf{p}}^{\mathbf{H}}}{\partial \mathbf{H}}$ is the Jacobian matrix, and it will be denoted by $\mathbf{J}$. Considering Eq. (6) in Eq. (5), we can estimate the error for a perturbation of the parameters

$$
\begin{aligned}
F(\mathbf{H}+\boldsymbol{\Delta} \mathbf{H}) & \approx\left(\mathbf{T}_{\mathrm{p}}^{\mathbf{H}}+\mathbf{J} \boldsymbol{\Delta} \mathbf{H}-\hat{\mathbf{T}}_{\mathrm{p}}\right)^{T}\left(\mathbf{T}_{\mathrm{p}}^{\mathbf{H}}+\mathbf{J} \boldsymbol{\Delta} \mathbf{H}-\hat{\mathbf{T}}_{\mathrm{p}}\right) \\
& =F(\mathbf{H})-2(\mathbf{J} \boldsymbol{\Delta} \mathbf{H})^{T}\left(\hat{\mathbf{T}}_{\mathrm{p}}-\mathbf{T}_{\mathrm{p}}^{\mathbf{H}}\right)+(\mathbf{J} \boldsymbol{\Delta} \mathbf{H})^{T} \mathbf{J} \boldsymbol{\Delta} \mathbf{H} \\
& =F(\mathbf{H})-\boldsymbol{\Delta} \mathbf{H}^{T}\left[2 \mathbf{J}^{T}\left(\hat{\mathbf{T}}_{\mathrm{p}}-\mathbf{T}_{\mathrm{p}}^{\mathbf{H}}\right)-\mathbf{J}^{T} \mathbf{J} \boldsymbol{\Delta} \mathbf{H}\right] .
\end{aligned}
$$

Thus, by identification, we deduce

$$
\frac{\partial F}{\partial \boldsymbol{\Delta} \mathbf{H}}(\mathbf{H}) \approx-2 \mathbf{J}^{T}\left(\hat{\mathbf{T}}_{\mathrm{p}}-\mathbf{T}_{\mathrm{p}}^{\mathbf{H}}\right)+\mathbf{J}^{T} \mathbf{J} \boldsymbol{\Delta} \mathbf{H} .
$$

We seek a perturbation $\boldsymbol{\Delta} \mathbf{H}$ that minimizes $F$, i.e. $\frac{\partial F}{\partial \boldsymbol{\Delta} \mathbf{H}}=\mathbf{0}$. Therefore Eq. (7) provides the expression

$$
\Delta \mathbf{H}=\left[\mathbf{J}^{T} \mathbf{J}\right]^{-1} \mathbf{J}^{T}\left(\hat{\mathbf{T}}_{\mathrm{p}}-\mathbf{T}_{\mathrm{p}}^{\mathbf{H}}\right) .
$$

Notice that calculation of $\boldsymbol{\Delta} \mathbf{H}$ only requires the Jacobian matrix and the Gauss-Newton method is less computational consuming when compared with Newton-Raphson technique, since for each iteration the thermal problem has to be solved $1+m$ times.

\subsection{The Levenberg-Marquardt method}

The Levenberg-Marquardt method is also a specific technique to solve nonlinear least squares problems where the parameters variation is an interpolation between the GaussNewton method and the gradient descent method. Levenberg replaced Eq. (8) by a damped version given as $[18$

$$
\Delta \mathbf{H}=\left[\mathbf{J}^{T} \mathbf{J}+\lambda \mathbf{I}\right]^{-1} \mathbf{J}^{T}\left(\hat{\mathbf{T}}_{\mathrm{p}}-\mathbf{T}_{\mathrm{p}}^{\mathbf{H}}\right),
$$

where $\lambda$ is a non-negative damping factor and $\mathbf{I}$ denotes the identity matrix. This factor provides a better stability and increases the admissible convergence basin where one can choose the initial condition. Notice that we recover the Gauss-Newton method with $\lambda=0$. The critical point consists in choosing the damping factor since too large values will reduce the efficiency of the method and dramatically increase the computational cost. Heuristic approaches to evaluate the the factor have been given in [18

To avoid slow convergence in the direction of small gradients, Marquardt [19] proposed to scale the components of the gradient substituting the identity matrix by a diagonal matrix with the elements of the diagonal of $\mathbf{J}^{T} \mathbf{J}$, and Eq. (9) becomes

$$
\boldsymbol{\Delta} \mathbf{H}=\left[\mathbf{J}^{T} \mathbf{J}+\lambda \operatorname{diag}\left(\mathbf{J}^{T} \mathbf{J}\right)\right]^{-1} \mathbf{J}^{T}\left(\hat{\mathbf{T}}_{\mathrm{p}}-\mathbf{T}_{\mathrm{p}}^{\mathbf{H}}\right) .
$$


There are several versions for the Levenberg-Marquardt method. The difference between them relies on the criterion for the convergence evaluation and on the evolution of the parameter $\lambda$. For this work, we accept the results of an iteration $n$ if the parameter $\rho^{n}$ is larger that a given tolerance $\epsilon$ 20. Hence,

$$
\rho^{n}=\frac{F(\mathbf{H})-F(\mathbf{H}+\boldsymbol{\Delta} \mathbf{H})}{2 \boldsymbol{\Delta} \mathbf{H}^{T}\left(\lambda^{n} \boldsymbol{\Delta} \mathbf{H}+\mathbf{J}^{T}\left(\hat{\mathbf{T}}_{\mathrm{p}}-\mathbf{T}_{\mathrm{p}}^{\mathbf{H}}\right)\right)} .
$$

To start this process a initial value for the damping factor $\lambda_{0}$ should be provide by the user. If the previous criterion is satisfied, the perturbation $\mathbf{\Delta H}$ is accepted and $\lambda$ is reduced till a limit value

$$
\lambda^{n+1}=\max \left[\frac{\lambda^{n}}{L_{\mathrm{dn}}} ; \lambda_{\min }\right] .
$$

Otherwise the set of parameters $\mathbf{H}$ remains unaltered, and $\lambda$ is increased

$$
\lambda^{n+1}=\min \left[\lambda^{n} L_{\mathrm{up}} ; \lambda_{\max }\right] .
$$

The evaluation of the terms of Eq. (10) follows the same procedure as Gauss-Newton method. Thus, the Levenberg-Marquardt method also need to solve the thermal problem $1+m$ times in each iteration.

\section{Synthetic tests}

We propose several synthetic benchmarks to assess the efficiency and robustness of the minimization methods. A set of parameter of reference is chosen to compute the reference solution. We then select 10 points on the upper and lower surfaces of the polymer we shall use in the identification algorithm. The first benchmark aims to compare the three minimization methods in terms of convergence and computational cost where we show that the Gauss-Newton method is the best compromise. The second benchmark deals with the basin of convergence. Random initial parameters are prescribed and we assess the method ability to recover the reference parameters.

\subsection{The manufactured reference solution}

To design the manufactured solution, we use $L_{\mathrm{in}}=0.17 \mathrm{~m}, L_{\mathrm{c}}=0.3 \mathrm{~m}, L_{\mathrm{out}}=0.13 \mathrm{~m}$, $t_{\mathrm{p}}=1.42 \times 10^{-3} \mathrm{~m}$, and $t_{\mathrm{c}}=0.012 \mathrm{~m}$. The material properties are $k_{\mathrm{p}}=0.17 \mathrm{~W} /(\mathrm{m} \mathrm{K})$, $k_{\mathrm{c}}=123 \mathrm{~W} /(\mathrm{m} \mathrm{K}), \rho_{\mathrm{p}}=1040 \mathrm{~kg} / \mathrm{m}^{3}$, and $c_{p_{\mathrm{p}}}=2050 \mathrm{~J} /(\mathrm{kg} \mathrm{K})$. The extrusion velocity is $0.0175 \mathrm{~m} / \mathrm{s}$, and the temperature on $\Gamma_{\text {sup }}$ is approximated by a polynomial function, based on the values measured by a set of thermocouples, which is given as

$$
T_{\text {sup }}=48180 x_{1}^{5}-45151 x_{1}^{4}+15502 x_{1}^{3}-2366.9 x_{1}^{2}+142.77 x_{1}+308.4, \quad x_{1} \in[0 ; 0.3] .
$$

For the remaining boundary conditions, it was defined $h_{\text {int }}=500 \mathrm{~W} /\left(\mathrm{m}^{2} \mathrm{~K}\right), h_{\mathrm{air}}=$ $50 \mathrm{~W} /\left(\mathrm{m}^{2} \mathrm{~K}\right), T_{\text {in }}=493 \mathrm{~K}\left(220^{\circ} \mathrm{C}\right)$, and $T_{\text {air }}=298.5 \mathrm{~K}\left(25.5^{\circ} \mathrm{C}\right)$.

As the set calibrator/polymer has a regular geometry, we use a structured quadrangular mesh as displayed in Fig. 5 where $N_{\mathrm{p}}, N_{\mathrm{c}}$, and $N_{\mathrm{h}}$ denote the number of elements on polymer and calibrator on $x_{2}$ direction and the number of elements on $x_{1}$ direction on calibrator, respectively. 


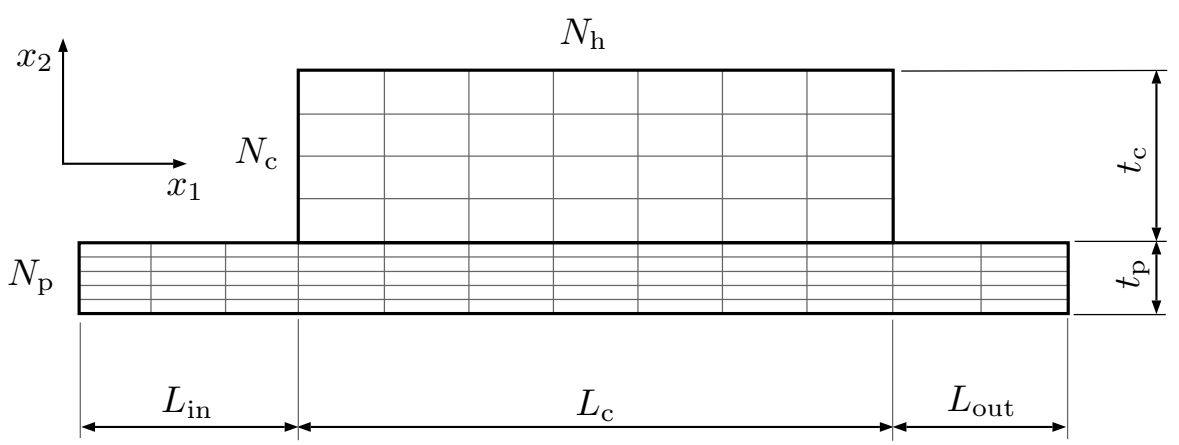

Figure 5: Schematic mesh for the polymer/calibrator model.

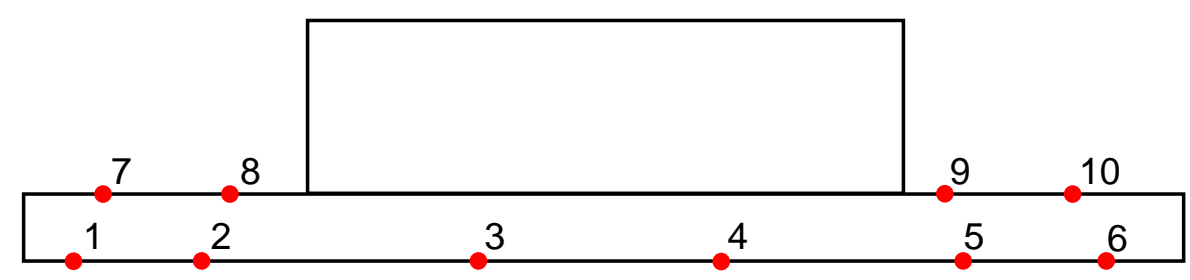

Figure 6: Representation of the measured points location.

Table 1: Coordinates and temperatures obtained numerically in the considered points.

\begin{tabular}{c|c|c|c}
\hline point & coordinate $x_{1}[\mathrm{~m}]$ & coordinate $x_{2}[\mathrm{~m}]$ & temperature $[\mathrm{K}]$ \\
\hline 1 & 0.045 & 0.0 & 466.6869 \\
2 & 0.081 & 0.0 & 456.1800 \\
3 & 0.221 & 0.0 & 418.5102 \\
4 & 0.395 & 0.0 & 355.3805 \\
5 & 0.471 & 0.0 & 339.6116 \\
6 & 0.587 & 0.0 & 327.1214 \\
7 & 0.103 & $1.42 \times 10^{-3}$ & 450.1617 \\
8 & 0.137 & $1.42 \times 10^{-3}$ & 441.3151 \\
9 & 0.485 & $1.42 \times 10^{-3}$ & 326.0922 \\
10 & 0.527 & & 328.0068 \\
\hline
\end{tabular}

The thermal model was evaluated with the given parameters and the temperature data was extracted at the 10 points given in Fig. 6] and reported in Table 1 (coordinates and temperatures). We define the dimensionless error estimator

$$
E_{n}=\sum_{i} \frac{2\left(H_{i}^{n+1}-H_{i}^{n}\right)}{H_{i}^{n+1}+H_{i}^{n}}
$$

and we stop the iterative procedure when the error between two consecutive iterations is lower than $1 \times 10^{-4}$. 


\subsection{Minimization methods comparison}

Convergence tests for the Newton-Raphson method, the Gauss-Newton method, and the Levenberg-Marquardt method are carried out to select the most performavit algorithm. For this benchmark, a set of four parameters is considered, namely: the heat transfer coefficient at the polymer/calibrator interface, the convection heat transfer coefficient of the air, the polymer inflow temperature, and the temperature of the air.

We proceed in the following way: all the parameters are set to the reference values except one that we set to $90 \%$ of the reference value. Computation is then carried out and we evaluate the number of iterations to reach the reference values. We then obtain four numerical tests where we successively perturbe $h_{\mathrm{int}}, h_{\mathrm{air}}, T_{\mathrm{in}}$, and $T_{\mathrm{air}}$. In Fig. 7 it is displayed the dimensionless error curves for the Newton-Raphson method. We notice that the method did not converge for the cases where $h_{\text {air }}$ and $T_{\text {air }}$ were perturbed, which disqualify the algorithm for future applications.
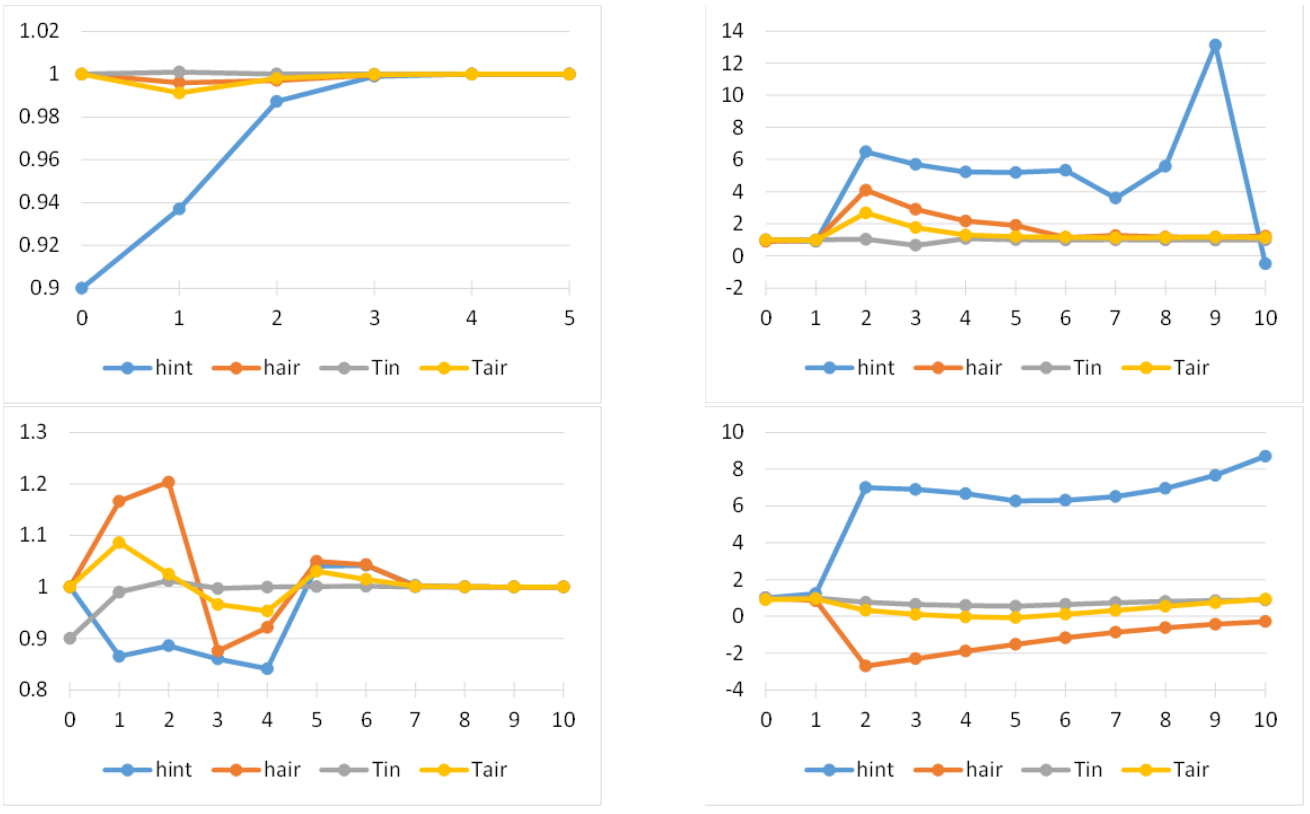

Figure 7: The evolution of the parameters during the optimization with Newton-Raphson with initial variations of $h_{\text {int }}$ (top left), $h_{\text {air }}$ (top right), $T_{\text {in }}$ (down left), and $T_{\text {air }}$ (down right).

We proceed in the same way with the Gauss-Newton technique and we report in Fig. 8 the error curves with respect to the number of iterations. Convergence is achieved in all the situations which qualifies the method to be used in more complex situations.

At last, the Levenberg-Marquardt method is tested for the same situations. The additional parameters required by the method are $\epsilon=1 \times 10^{-2}, \lambda_{\min }=1 \times 10^{-7}$, $\lambda_{\max }=1 \times 10^{7}, L_{\mathrm{dn}}=9$, and $L_{\mathrm{up}}=11$. It is given in Fig. 9 the error curves for the four perturbations and observe that the algorithm converged in all the cases.

We report in Table 2 the number of iterations and time consuming (in seconds) for each test. The Newton-Raphson technique is clearly the worst algorithm whereas the Gauss-Newton and Levenberg-Marquardt methods are faster and converged in all tests. 

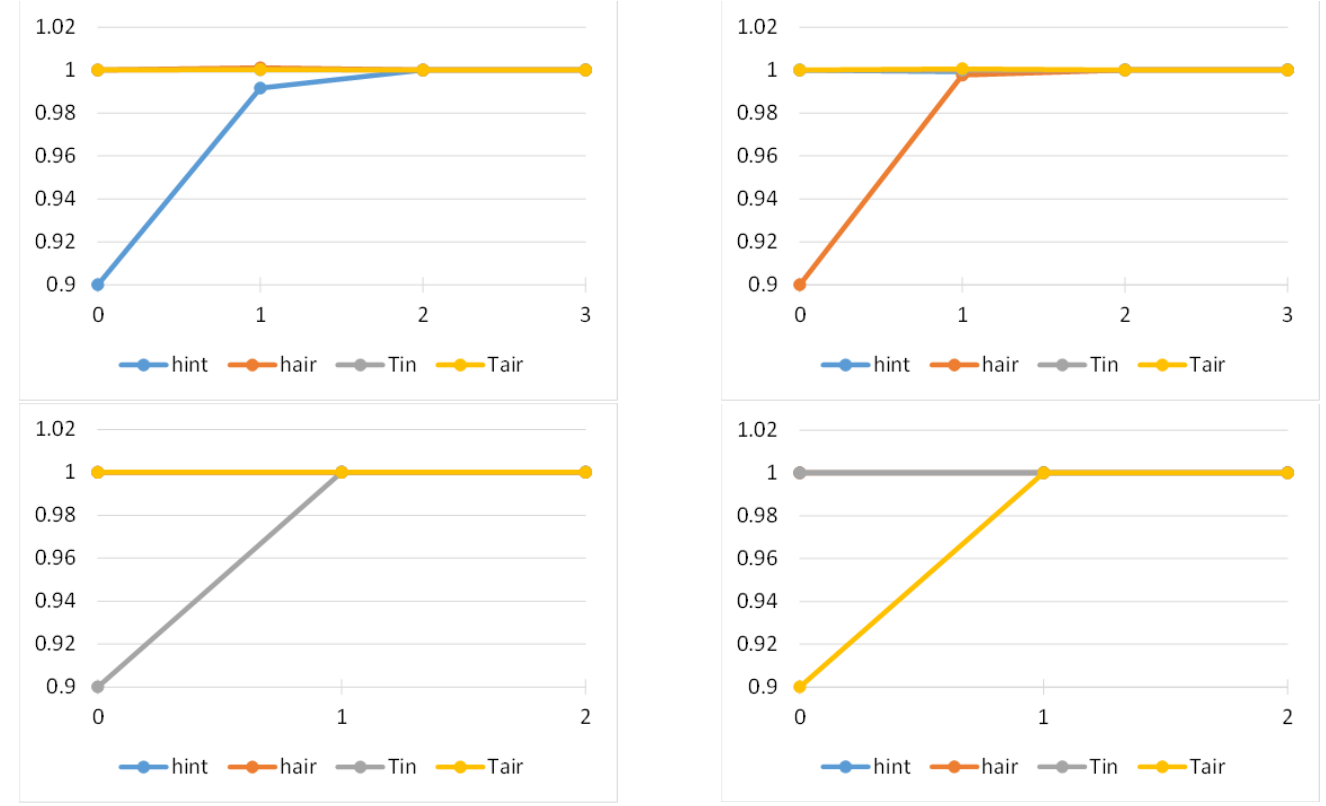

Figure 8: The evolution of the parameter during the optimization with Gauss-Newton with initial variations of $h_{\text {int }}$ (top left), $h_{\text {air }}$ (top right), $T_{\text {in }}$ (down left), and $T_{\text {air }}$ (down right).
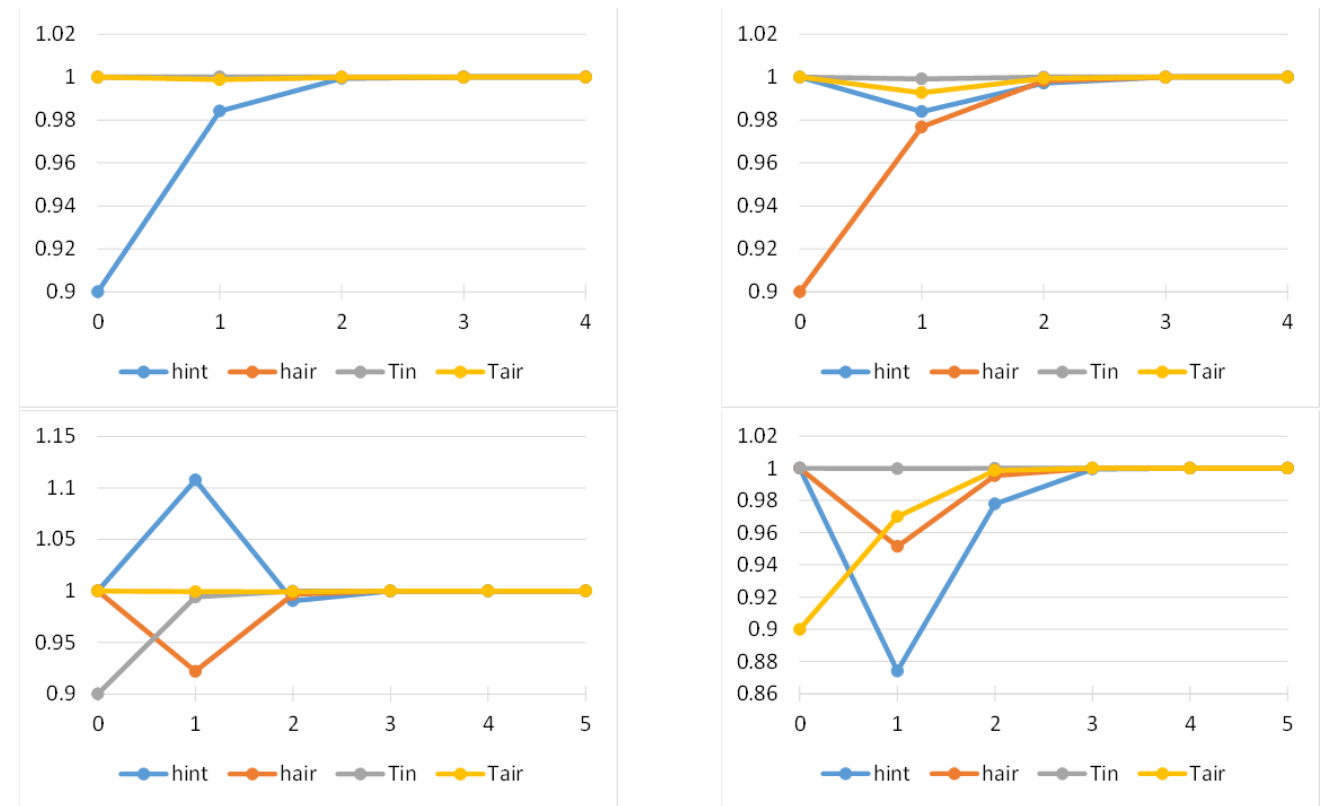

Figure 9: The evolution of the parameter during the optimization with Levenberg-Marquardt with initial variations of $h_{\text {int }}$ (top left), $h_{\text {air }}$ (top right), $T_{\text {in }}$ (down left), and $T_{\text {air }}$ (down right). 
On the other hand, the Gauss-Newton method is more efficient with less iterations to converge for the correct solution. So, we will adopt the Gauss-Newton algorithm to carry out all the following benchmarks and tests.

\begin{tabular}{|c|c|c|c|c|c|c|c|c|c|c|}
\multicolumn{10}{c|}{ Table 2: Summary of the presented tests. } \\
\cline { 2 - 12 } \multicolumn{1}{c|}{} & \multicolumn{2}{|c|}{$h_{\text {int }}$} & \multicolumn{2}{|c|}{$h_{\text {air }}$} & \multicolumn{2}{|c|}{$T_{\text {in }}$} & \multicolumn{2}{|c|}{$T_{\text {air }}$} & \multicolumn{2}{c|}{ all } \\
\cline { 2 - 12 } & iter & time & iter & time & iter & time & iter & time & iter & time \\
\hline NR & 5 & 1714.1 & - & - & 10 & 3514.3 & - & - & 5 & 1581.25 \\
GN & 3 & 142.89 & 3 & 129.62 & 2 & 88.409 & 2 & 86.018 & 4 & 169.63 \\
LM & 4 & 177.11 & 4 & 170.02 & 5 & 246.11 & 5 & 259.58 & 5 & 250.36 \\
\hline
\end{tabular}

\subsection{Test with a control parameter}

Beyond the parameters studied in the previous tests, the extrusion velocity will also be included in the identification process. Although this parameter is well defined during the experimental activity, it is used as a control parameter to validate the results. Indeed, since the parameters identification algorithm provides an approximation of the velocity that we compare with the measured (or prescribed) one, we obtain an estimate of the approximation accuracy. Consequently, the velocity has not the status of a unknown parameter but acts as a control parameter which assess the solution quality.

In order to evaluate the basin of convergence, $i$. $e$. the domain where we can choose the initial condition which guarantees the convergence of the iterative sequence, we choose the initial guess in an interval of $\pm 50 \%$ of the reference parameter for $h_{\text {int }}, h_{\text {air }}$, and $u$, and the temperatures (in Kelvin) ranges in a $\pm 10 \%$ interval around the reference temperatures. Therefore, the dimensionless value of each parameters for initial approximation can vary between 0.5 and 1.5 or 0.9 and 1.1. Ten random sets of parameters belonging to the admissible initial domain have been chosen ( $c f$. Table 3). The additional information shows whether the control parameter coincides with the reference one or not. Only one negative case is reported and we underline that convergence is achieved with at most 5 iterations.

Table 3: Initial approximations for the performed tests and numbers of iterations to reach convergence.

\begin{tabular}{c|cccccccccc}
\hline test & 1 & 2 & 3 & 4 & 5 & 6 & 7 & 8 & 9 & 10 \\
\hline$h_{\text {int }}$ & 0.635 & 0.936 & 1.156 & 1.273 & 0.732 & 0.805 & 1.330 & 1.127 & 0.609 & 0.963 \\
$h_{\text {air }}$ & 1.205 & 1.464 & 0.879 & 1.403 & 1.143 & 1.223 & 1.047 & 0.872 & 0.900 & 1.185 \\
$T_{\text {in }}$ & 0.954 & 1.065 & 0.987 & 0.932 & 1.019 & 0.984 & 0.967 & 1.087 & 0.957 & 0.969 \\
$T_{\text {air }}$ & 0.918 & 1.059 & 1.079 & 1.028 & 0.969 & 1.008 & 0.937 & 1.043 & 0.996 & 0.905 \\
$u$ & 1.221 & 1.354 & 1.256 & 0.828 & 0.622 & 0.643 & 1.436 & 1.050 & 1.201 & 0.563 \\
\hline convergence & yes (5) & yes (4) & yes (5) & yes (6) & yes (5) & yes (5) & yes (5) & yes (4) & yes (5) & no \\
\hline
\end{tabular}

\section{Experimental case study}

The procedures proposed in this work, to find the optimal parameters that meet the experimental data, are now tested in a real situation, with data obtained with the 
prototype system, conceived to measure the heat transfer coefficient at the polymercalibrator interface [1]. For that purpose the prototype system was operated under specific process conditions and the average temperature at several points, identified in Fig. 6, was registered after achieving steady state conditions, during a measurement period of $20 \mathrm{~min}$. Regarding the fitting approach, in all tests performed in this section the Gauss-Newton method was considered, since it was the one that showed the best performance on the previous studies, described in the previous section.

The location of the measured points as well as the system properties, closely follows the data used in the synthetic tests ( $c f$. Table 1). During the experiments the air temperature was measured, $T_{\text {air }}=293 \mathrm{~K}$, and the profile velocity $u=0.0175 \mathrm{~m} / \mathrm{s}$ was imposed. Table 4 contains the average temperature values for all points, collected during the experimental run.

Table 4: Temperatures obtained on the experimental test.

\begin{tabular}{c|c}
\hline point & temperature $[\mathrm{K}]$ \\
\hline 1 & 477.31 \\
2 & 468.87 \\
3 & 441.81 \\
4 & 362.40 \\
5 & 342.02 \\
6 & 331.14 \\
7 & 463.98 \\
8 & 455.32 \\
9 & 329.97 \\
10 & 331.29 \\
\hline
\end{tabular}

The first parameter fitting test $(2+2$ points) followed the procedure proposed in 1], which consists of using the temperature of points 7 and 8 (see Fig. 6) to identify $h_{\text {air }}$ and $T_{\mathrm{in}}$, and the temperatures of points 9 and 10, to obtain $h_{\text {int }}$, as illustrated in Fig. 10.

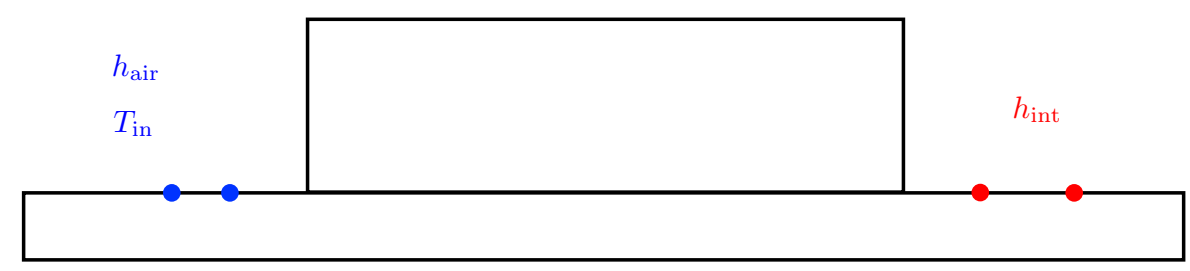

Figure 10: points used for the $2+2$ points fitting test.

The results obtained in this first test, given in Table 5 show the values obtained for the error Functional, $F=74.2 \mathrm{~K}^{2}$, for the error standard deviation, $\sigma=2.72 \mathrm{~K}$.

The main advantage of the new proposed fitting methodology is the ability of allowing the identification of the required parameter set that minimizes the error functional with any number of data points, providing that it is larger than the number of parameters to identify. This is expected to provide a better, i.e. more suitable overall, result when there is enough data measured during the experimental tests. The parameter values 
Table 5: Results obtained on the $2+2$ points fitting test.

\begin{tabular}{c|c}
\hline parameter & value \\
\hline$h_{\text {int }}$ & 903.21 \\
$h_{\text {air }}$ & 36.374 \\
$T_{\text {in }}$ & 498.00 \\
\hline$F$ & 74.2 \\
$\sigma$ & 2.72 \\
\hline
\end{tabular}

obtained on the second fitting (10 points) test, where all the available temperature data points were used, are given in Table 6. As shown, by the reduction of circa $30 \%$ and $60 \%$, respectively for $F$ and $\sigma$, when compared with the ones of the previous fit, this approach provided better results.

Table 6: Results obtained on the 10 points fitting test.

\begin{tabular}{c|c}
\hline parameter & value \\
\hline$h_{\text {int }}$ & 891.40 \\
$h_{\text {air }}$ & 32.920 \\
$T_{\text {in }}$ & 495.29 \\
\hline$F$ & 50.2 \\
$\sigma$ & 2.24 \\
\hline
\end{tabular}

Additional insights of both fitting procedures can be provided by the analysis of the temperature errors obtained after the fitting procedure, shown in Table 7 . On the case of the $2+2$ points fitting, since on the initial step just two points are used (points 7 and 8, cf. Fig.s 10 and 6), a perfect fit is obtained at those points, with null temperature errors. Moreover, the temperature errors obtained for the $2+2$ points fitting at points 9 and 10 is also lower than the ones obtained for the 10 points counterpart. This is again a consequence of the local incidence of the $2+2$ point fitting procedure. The main advantages of the 10 point fitting is clear when temperature errors on the remaining points (points 1-6) are analyzed. Since the 10 points fitting procedure considers simultaneously all the measured points the temperature errors obtained at points 1-6 is lower, especially in point 3 , thus providing an overall best performance.

The results obtained in this section clearly evidence the advantages of using the proposed approach with the propotype system developed for that purpose, to obtain the value of the heat transfer coefficients

\section{Conclusion}

In this work a novel fitting methodology to identify some unknown heat transfer coefficients of the thermoplastic profile extrusioncalibration stage, namely the interface polymer-calibrator and the air convection, was proposed. The fitting procedure employs experimental data collected with a previously developed experimental prototype system [1]. The novel methodology required the development of a modeling code for the heat transfer process, which involves discontinuous temperature and velocity fields and discontinuous materials properties. For this purpose a new second-order finite volume 
Table 7: Test with $h_{\text {int }}, h_{\text {air }}$ and $T_{\text {in }}$ with 10 points.

\begin{tabular}{c|c|c|c|c|c}
\hline \multirow{2}{*}{ point } & \multirow{2}{*}{ exp. temp. [K] } & \multicolumn{2}{|c}{$2+2$ points } & \multicolumn{2}{|c}{10 points } \\
\cline { 3 - 6 } & 477.31 & 480.01 & +2.70 & 476.67 & -0.64 \\
\hline 1 & 468.87 & 469.83 & +0.96 & 469.01 & +0.14 \\
2 & 441.81 & 432.50 & -9.30 & 437.89 & -3.92 \\
3 & 362.40 & 363.47 & +1.07 & 363.74 & +1.34 \\
4 & 342.02 & 345.67 & +3.65 & 345.38 & +3.36 \\
5 & 331.14 & 331.19 & +0.05 & 331.34 & +0.20 \\
6 & 463.98 & 463.98 & 0.00 & 464.55 & +0.57 \\
7 & 455.32 & 455.32 & 0.00 & 457.89 & +2.57 \\
8 & 329.97 & 329.46 & -0.51 & 326.31 & -3.66 \\
9 & 331.29 & 331.84 & 0.55 & 330.32 & -0.97 \\
\hline
\end{tabular}

method scheme was implemented. It is based in a cell-to-vertex reconstruction, being the vertex values computed via linear combinations of the closest cell values, where the coefficients are determined by a functional minimization. For the heat transfer coefficient identification (fitting) phase some alternative methodologies were assessed, being the Gauss-Newton method the one that showed be best efficiency in terms of calculation time and stability. Finally, the proposed methodology was tested with experimental data, which was also used to compare its performance with the one of the previous employed approach. The results obtained evidenced clearly the advantages of the newly proposed procedure.

\section{Acknowledgements}

This research was financed by FEDER Funds through Programa Operacional Factores de Competitividade - COMPETE and by Portuguese Funds through FCT — Fundação para a Ciência e a Tecnologia, within the Projects PEst-OE/MAT/UI0013/2014, PTDC/MAT/121185/2010, and UID $/ \mathrm{CTM} / 50025 / 2013$. The second author was also financed by project FCTANR/MAT-NAN/0122/2012.

\section{References}

[1] O.S. Carneiro, J.M. Nóbrega, A.R. Mota, C. Silva, Prototype and methodology for the characterization of the polymer-calibrator interface heat transfer coefficient, Polymer Testing, 32 (6) (2013) 1154-1161.

[2] F. Marques, S. Clain, G.J. Machado, B. Martins, O.S. Carneiro, J.M. Nóbrega, A New Energy Conservation Scheme for the Numeric Study of the Heat Transfer in Profile Extrusion Calibration, submitted (preprint available in https://hal.archives-ouvertes.fr/hal-01176796).

[3] C. Rauwendaal, Polymer Extrusion 5E, Hanser (2014).

[4] T. Sastrohartono, Y. Jaluria, M. Esseghir, V. Sernas, A numerical and experimental study of the three-dimensional transport in the channel of an extruder for polymeric materials, Int. J. Heat Mass Transfer, 36 (1995) 1957-1973.

[5] E. Mitsoulis, J. Vlachopoulos, F. A. Mirza, Finite element analysis of flow through dies and extruders channels, SPE ANTEC Tech. Papers, 30.53-58 (1984).

[6] M. Gupta, T. Kown, Y. Jaluria, Multivariant finite element for the three-dimensional simulation of viscous incompressible flows, Int. J. Numer. Meth. Fluids, 14 (1992) 557-585. 
[7] Y. Yu, X. Luo, Estimation of heat transfer coefficients and heat flux on the billet surface by an integrated approach, Int. J. Heat Mass Transfer, 90 (2015) 645-653.

[8] M.V. Murashov, S.D. Panin, Numerical modelling of contact heat transfer problem with work hardened rough surfaces, Int. J. Heat Mass Transfer, 90 (2015) 72-80.

[9] H. Versteeg, W. Malalasekera, An Introduction to Computational Fluid Dynamics: The Finite Volume Method 2E, Pearson, Prentice Hall (2007).

[10] C.-L. Xiao, H.-X. Huang, Optimal design of heating system for rapid thermal cycling mold using particle swarm optimization and finite element method, Appl. Therm. Eng., 64 (2014) 462-470.

[11] A. Agazzi, V. Sobotka, R. LeGoff, Y. Jarny, Optimal cooling design in injection moulding process - A new approach based on morphological surfaces, Appl. Therm. Eng., 52 (2013) 170-178.

[12] S.-C. Xue, G.W. Barton, Implementation of boundary conditions and global mass conservation in pressure-based finite volume method on unstructured grids forfluid flow and heat transfer simulations, Int. J. Heat Mass Transfer, 55 (2012) 5233-5243.

[13] E.O. Reséndiz-Flores, I.D. García-Calvillo, Application of the finite pointset method to nonstationary heat conduction problems, Int. J. Heat Mass Transfer, 71 (2014) 720-723.

[14] E.O. Reséndiz-Flores, F.R. Saucedo-Zendojo, Two-dimensional numerical simulation of heat transfer with moving heat source in welding using the finite pointset method, Int. J. Heat Mass Transfer, 90 (2015) 239-245.

[15] W. Uffrecht, B.Heinschke A. Günther, V. Caspary, S. Odenbach, Measurement of heat transfer coefficients at up to $25,500 \mathrm{~g}$ - A sensor test at a rotating free disk with complex telemetric instrumentation, Int. J. Therm. Sci., 96 (2015) 331-344.

[16] R. Costa, S. Clain, G.J. Machado, New cell-vertex reconstruction for finite volume scheme: Application to the convection-diffusion-reaction equation, Computers and Mathematics with Applications, 68 (2014) 1229-1249.

[17] R. Costa, S. Clain, G.J. Machado, Finite Volume Scheme Based on Cell-Vertex Reconstructions for Anisotropic Diffusion Problems with Discontinuous Coefficients, 14th International Conference in Computational Science and Its Applications - ICCSA 2014, Lecture Notes in Computer Science, 8579 (2014) 87-102.

[18] K. Levenberg, A Method for the Solution of Certain Problems in Least Squares, Quart. Appl. Math., 2 (1944) 164-168.

[19] D. Marquardt, An Algorithm for Least-Squares Estimation of Nonlinear Parameters, SIAM J. Appl. Math., 11 (1963) 431-441.

[20] H.B. Nielson, Damping Parameter In Marquardt's Method, Technical Report IMM-REP-1999-05, Dept. of Mathematical Modeling, Technical University Denmark. 